\title{
News from Somewhere: William Morris et l'héritage socialiste britannique
}

News from Somewhere: William Morris and the British Socialist Heritage

\section{Michel Prum}

\section{(2) OpenEdition}

1 Journals

Édition électronique

URL : http://journals.openedition.org/rfcb/3248

DOI : $10.4000 /$ rfcb.3248

ISSN : 2429-4373

Éditeur

CRECIB - Centre de recherche et d'études en civilisation britannique

\section{Édition imprimée}

Date de publication : 1 septembre 2004

ISBN : 2-911580-19-2

ISSN : 0248-9015

\section{Référence électronique}

Michel Prum, « News from Somewhere: William Morris et l'héritage socialiste britannique », Revue

Française de Civilisation Britannique [En ligne], XIII-1 | 2004, mis en ligne le 01 septembre 2004, consulté le 07 octobre 2019. URL : http://journals.openedition.org/rfcb/3248 ; DOI : 10.4000/rfcb.3248

Ce document a été généré automatiquement le 7 octobre 2019.

\section{cc)}

Revue française de civilisation britannique est mis à disposition selon les termes de la licence Creative Commons Attribution - Pas d'Utilisation Commerciale - Pas de Modification 4.0 International. 


\title{
News from Somewhere: William Morris et l'héritage socialiste britannique
}

\author{
News from Somewhere: William Morris and the British Socialist Heritage
}

Michel Prum

1 Les Nouvelles de Nulle Part viennent de quelque part, comme toute œuvre littéraire : c'est une évidence que l'on voudra bien me pardonner de reformuler ici. On pense bien sûr à Ruskin, à bien des égards le maître à penser, et, dans une moindre mesure, à Carlyle. On sait également l'enthousiasme que provoqua en Morris la lecture de l'Utopie (1516) de Thomas More. Des livres entiers ont été écrits sur son médiévisme, nourri des romans de Walter Scott dès l'âge de quatre ans, puis par des ouvrages historiques (ceux de l'école d'Oxford, en particulier) ou des textes médiévaux. E.P. Thompson montre aussi l'influence de Keats, les derniers mots de News from Nowhere : 'It may be called a vision rather than a dream' renvoyant aux vers de l'ode to a Nightingale: 'Was it a vision, or a waking dream? / Fled is that music: - do I wake or sleep?'1 Enfin l'influence du marxisme n'est plus à démontrer. On se reportera à l'étude très complète que Paul Meier a réalisée dans La Pensée utopique de William Morris ${ }^{2}$. Il serait inutile de reprendre ces chemins déjà explorés. En revanche, il me semble que Paul Meier est incomplet lorsqu'il parle des «pré-marxistes" britanniques - je n'emploierai d'ailleurs pas cette expression, un peu trop téléologique: les "pré-marxistes» (comme les "présocratiques ») attendant l'arrivée de Marx (ou de Socrate) pour se réaliser pleinement ! 3

\section{Amnésie ou mémoire ouvrière}

En Grande-Bretagne, une faille semble scinder la pensée socialiste du XIX ${ }^{\mathrm{e}}$ siècle. Dans la première moitié du siècle, dans les années 1820 et 1830 en particulier, cette pensée est riche, voire bouillonnante, et les ouvrages fondamentaux se succèdent, élaborant une réflexion très novatrice, qui sera, aux côtés de l'économie politique classique (Smith, Ricardo) et de l'hégelianisme, la troisième source de la pensée marxienne ${ }^{4}$. 
Cette période voit la parution de The Distribution of Wealth (1824) et The Appeal of One Half the Human Race (1825) de William Thompson, de Labour Defended against the Claims of Capital (1825) de Thomas Hodgskin, de la Lecture on Human Happiness (1826) de John Gray, ou encore du très fécond Labour's Wrongs and Labour's Remedy (1839) de John Francis Bray, qui retiendra particulièrement mon attention dans cet article. A cette créativité théorique correspond l'essor des luttes ouvrières, culminant avec la grande manifestation chartiste de 1848. C'est l'époque où, qu'on la souhaite ou qu'on la craigne, beaucoup s'imaginent proche une grande révolution sociale. "Pour l'Angleterre, écrit Engels dans le numéro de la Gazette rhénane daté du 10 décembre 1842 , cette révolution est inévitable » et Carlyle s'étonne en 1839 que la révolution n'ait pas encore éclaté, alors que les travailleurs sont restés huit ans assis au festin du Barmécide ${ }^{5}$. La fin de siècle - la fin des années 1880 et les années 1890 en particulier est également une période de vive agitation ouvrière. On pense à la grève historique des dockers de Londres en 1889, paralysant le plus grand port du monde durant un mois, ou à l'explosion des effectifs syndicaux, ou encore à l'agitation politique qui conduira quelques années plus tard à la naissance du Parti travailliste.

Entre les deux périodes : rien, ou presque. Comme le note E.P. Thompson : 'In the years between 1870 and 1880 (and even for ten years before 1870) no consistent Socialist propaganda - not even of a dozen or twenty members - had existed in Britain. ${ }^{6}$ Dans cet étrange vide politique, "la flamme du socialisme semble presque éteinte ", comme le remarque François Bédarida ${ }^{7}$. Les organisations ouvrières se sont très massivement ralliées au libéralisme, dans ce qu'on a appelé l'alliance Lib-Lab, et «l'idole des travailleurs, c'est Gladstone $»^{8}$. Marx, en 1878 , note amèrement que la classe ouvrière britannique « n'est plus rien d'autre que la queue du grand Parti libéral, c'est-à-dire de ses oppresseurs, les capitalistes ${ }^{\prime}$. 'Working men had ceased to think', tranche le poète chartiste Thomas Cooper ${ }^{10}$ et Kropotkine se sent si seul dans cette Angleterre atone qu'il compare dans ses Mémoires Londres à un tombeau, auquel il préfère la France, dût-il y être arrêté.

4 William Morris rédige ses Nouvelles de Nulle Part durant cette seconde période d'agitation ouvrière. Mais il écrit dans le pays où militèrent non seulement Robert Owen, mais tous les 'early Socialists' que j'ai précédemment cités. Mon hypothèse est que l'on ne peut faire comme si la première période d'agitation socialiste n'avait pas existé et qu'il n'était resté trace, à l'époque de News from Nowhere, que de Robert Owen et des utopistes français. C'est pourtant l'impression que donne Paul Meier dans l'ouvrage déjà cité, qui consacre des pages à Babeuf, Fourier, Louis Blanc, mais ne signale même pas le nom des socialistes britanniques ${ }^{11}$. Pourtant William Thompson, par exemple, fut lui aussi un très grand nom du mouvement coopératiste, le seul capable de tenir tête à Robert Owen, et son engagement féministe lui a donné une certaine notoriété au $\mathrm{XX}^{\mathrm{e}}$ siècle. Morris les a-t-il lus ? Il est sinon probable, du moins possible qu'il en ait lu certains. Labour's Wrongs, de John Francis Bray, devint un classique de la littérature ouvrière et fut réédité à plusieurs reprises, en particulier dans une édition partielle, en 1868. Morris a pu le lire, ou du moins en entendre parler, ne serait-ce que dans les ouvrages de Marx, qui le cite très abondamment: il en traduit ainsi neuf pages dans Misère de la philosophie ${ }^{12}$. Dans sa deuxième partie, consacrée aux "sources et influences" de Morris, Paul Meier prend soin d'annoncer, à propos des socialistes utopistes qui ont précédé Morris : "Il peut aussi ne pas y avoir de lecture du tout, ce qui n'exclut nullement la possibilité d'influence. Les ceuvres de ces socialistes utopiques pouvaient à 
maintes reprises être évoquées dans les fréquentes conversations et discussions qu'avait Morris avec ses compagnons de lutte. Leurs idées étaient 'dans l'air', et Morris était un auditeur attentif ${ }^{\prime 13}$. La question est de savoir si les socialistes britanniques des années 1820 ou 1830 étaient encore «dans l'air » à l'époque de News from Nowhere. Nous aurions de la peine à croire qu'aucune transmission ne se soit faite dans les milieux ouvriers, d'une génération à l'autre, et que l'amnésie ait été totale. Et, redisons-le, Marx pouvait aider Morris, si besoin était, à opérer cette « anamnèse ouvrière ».

\section{L'homme nouveau et la chrysalide}

5 Paul Meier, dans son ouvrage sur William Morris, souligne de façon récurrente « l'un des aspects fondamentaux de la pensée utopique de Morris, peut-être même... son aspect essentiel, celui qui en révèle le mieux la maturité et la portée $»^{14}$. Quelle est cette avancée conceptuelle ? C'est ce que Meier appelle la théorie des deux stades, celle qu'expose Marx dans sa Critique du programme de Gotha (écrite en 1875, publiée seulement en 1891, et que, selon Meier, Morris aurait pu connaitre oralement par ses contacts avec Engels, qui en détenait le manuscrit). Meier déclare :

Alors que les socialistes anglais $\mathrm{du}_{\mathrm{XIX}}{ }^{\mathrm{e}}$ siècle se partageaient en général entre l'opportunisme et l'anarchisme, les uns rêvant d'une lente imprégnation des institutions actuelles ou de l'instauration d'un socialisme d'État rigoureux et définitif, les autres imaginant l'établissement immédiat et violent d'un égalitarisme absolu, seul William Morris, à la suite de Marx et d'Engels, sut exposer alors cette même théorie qui devait ouvrir la perspective historique des partis marxistes au $\mathrm{XX}^{\mathrm{e}}$ siècle, la théorie des deux stades ${ }^{15}$.

6 Le premier stade, le stade du "socialisme ", est celui qui suit immédiatement la prise de pouvoir par le prolétariat et correspond au mot d'ordre: "de chacun selon ses capacités, à chacun selon son travail »; c'est un stade où règne encore l'inégalité. Le second stade, beaucoup plus tardif, celui du « communisme», permettra à la société d' « inscrire sur ses drapeaux »: «de chacun selon ses capacités, à chacun selon ses besoins ». Cette double succession historique apparaît clairement dans l'important chapitre XVII : 'How the change came', dans le long récit que fait le vieux Hammond du processus révolutionnaire qui débute en 1952. Pourquoi cette première étape « socialiste» avant l'accession au « communisme»? Dans True and False Society (1886), Morris explique qu'il faudra "une période de transition au cours de laquelle les hommes se débarrasseront des habitudes de pensée entretenues par de longs siècles de tyrannie et de concurrence commerciale et apprendront qu'il est de l'intérêt de chacun que tous connaissent la prospérité $»^{16}$.

7 Cette période de transition permettra de créer ce que l'on appellera plus tard, avec la révolution cubaine, "l'homme nouveau" (el ombre nuevo), capable de vivre dans le monde "communiste» de véritable égalité. En effet, 'the once-poor had such a feeble conception of the real pleasure of life ${ }^{17}$ : ils ne pourraient profiter pleinement de la société nouvelle. Pour cette seconde phase, Morris parle de "nouvelle naissance »: 'I was, as it were, really new-born', ${ }^{18}$ estime le narrateur lors de son équipée à la rame sur la Tamise, et le vieux Hammond, à la fin de sa description de la Révolution, commente : 'The world was being brought to its second birth' ${ }^{19}$ A cette seconde naissance correspond une " seconde enfance ": 'the second childhood of the world', ${ }^{20}$ dit Hammond.

8 Cette notion de phase de transition permettant à l'homme de se transformer et de devenir digne du stade suprême de la société égalitaire n'est pas nouvelle dans la 
pensée socialiste britannique au moment où Morris écrit sa 'romance'. Dans l'ouvrage de John Francis Bray que nous avons mentionné, Labour's Wrongs and Labour's Remedy (1839), l'auteur entrevoit déjà le problème. Abîmé comme il l'est par le monde actuel, l'homme n'est pas capable de vivre pleinement la société idéale.

Every step to the establishment of a better system must be made by those who have grown up under the present system and who are, therefore, more or less imbued with the depravity and ill-feelings which this system generates... Some preparatory steps must be discovered and made use of - some movement partaking partly of the desired system - some intermediate resting place, to which society may go with all its follies, and from which it may move forward, imbued with those qualities and attributes without which the system of community and equality cannot as such have existence. ${ }^{21}$

Pour Bray, le communisme ('the system of community') ne peut être atteint qu'au travers de cet 'intermediate resting place'. Ce " havre » donnerait aux êtres humains une maturité suffisante et permettrait aussi à la société d'accéder à l'abondance économique. Le stade de transition créerait 'a race of human beings widely different in character and habits from those who now exist'. ${ }^{22}$ Cette "nouvelle race » fait penser à la nouvelle humanité du XXII ${ }^{e}$ siècle britannique décrite par Morris. Bray ne parle pas, comme Morris, de «nouvelle naissance », mais il emploie la métaphore de la chrysalide, stade obligé pour le ver qui veut se transformer en papillon. Certes, plus de cinquante ans avant les Nouvelles de Nulle Part, Bray ne pouvait donner au problème de la transition vers le communisme toute la complexité et la subtilité auxquelles Morris, sans doute aidé par Engels, pourra accéder. Mais nous sommes dans le même registre de la transformation et du dépassement vers une meilleure nature humaine.

La «nature humaine » est, on le sait, une notion qui faisait bondir Morris. Pour lui, la nature humaine n'existe pas : elle n'est que « l'ensemble des rapports sociaux » comme l'écrit Marx dans sa sixième thèse sur Feuerbach ${ }^{23}$. 'Human nature!', s'exclame le vieux Hammond, 'what human nature? The human nature of paupers, of slaves, of slave-holders, or the human nature of wealthy freemen? ${ }^{24}$ Sans bien sûr formuler cette notion en termes de " rapports sociaux ", Bray n'est pas loin de cette idée lorsqu'il dit que si l'on échangeait un bébé éthiopien et un bébé anglais, on obtiendrait le caractère et les opinions de l'Éthiopien chez le petit Anglais et vice-versa ${ }^{25}$. Mais ici, Bray comme Morris s'inscrivent dans une tradition beaucoup plus longue, qui remonte aux philosophes des Lumières, en particulier à Helvétius, en passant par Godwin et Owen. L'homme est le produit des circonstances. 'From the nature of man, he must always be the creature of circumstances', note Bray ${ }^{26}$. La nature de l'homme est de ne pas avoir de nature, pourrait-on presque dire.

\section{L'œuvre des travailleurs eux-mêmes}

Cette Révolution en deux temps, vers le socialisme puis le communisme, qui enfantera l'homme nouveau, Morris pense, en bon marxiste, qu'elle sera menée à bien par le prolétariat. Dans les statuts de la Socialist League, Morris reprend la phrase de Marx: "l'émancipation des travailleurs sera l'œuvre des travailleurs eux-mêmes". Ce rôle du prolétariat apparaît dans le chapitre 'How the change came'. Les dirigeants bourgeois radicaux qui se prétendent proches des ouvriers se retrouvent, dans la lutte, du mauvais côté de la barrière. Morris leur réserve ses sarcasmes. Dans le récit du processus révolutionnaire, Hammond s'en prend tout particulièrement à un journal 
libéral qui, 'after a preamble in which it declared its undeviating sympathy with the cause of labour', explique que la façon la plus humaine ('merciful') de traiter les révolutionnaires pauvres et insensés est 'to shoot them at once, so as to stop others from drifting into a position in which they would run a chance of being shot'. ${ }^{27}$

Cette idée n'est certes pas présente chez Robert Owen, dont la volonté de sauver les classes ouvrières relève du paternalisme. Mais les socialistes de la première moitié du siècle ne sont pas tous sur ces bases. John Francis Bray estime que l'erreur des travailleurs est d'avoir confié leur destin à des hommes politiques issus d'autres classes sociales. Il pense sans doute aux dirigeants bourgeois qui menèrent la classe ouvrière en bateau lors de la grande duperie de 1832, ou encore aux leaders de l'Anti-Corn-Law League qui dévoyèrent la mobilisation ouvrière au profit du libre échange. Ces personnages, 'from their position in society... were necessarily weak champions and blind guides $^{28}$. Leur position sociale est donc le facteur déterminant qui les discrédite. C'est aux travailleurs qu'il appartient de se libérer. 'The producers have but to make an effort and by them must every effort for their own redemption be made', souligne-t-il ${ }^{29}$. Comme le note A.L. Morton: 'Bray also grasped more firmly than his predecessors and many of his successors the truth that a change in the condition of the workers must be effected by their own efforts ${ }^{30}$. L'idée du rôle moteur de la classe ouvrière n'est donc pas une idée neuve dans l'Angleterre de la seconde moitié du siècle.

Robert Owen fait partie de ces «prédécesseurs » dont parle Morton, incapables de voir ce rôle moteur. William Morris, tout en louant le philanthrope de New Lanark, critique violemment l'owenisme. Paul Meier voit même en Morris, pourrait-on dire, «l'antiOwen». L'utopie morrissienne est pour lui «la négation» de l'owenisme ${ }^{31}$. Il cite un texte paru en 1885 dans Commonweal dans lequel Morris explique qu'il est impossible "d'établir une véritable communauté socialiste au milieu de la société capitaliste, un îlot social au milieu d'un océan d'individualisme $»^{32}$. Le village coopératiste owenien est pour Morris une voie de garage. C'est un leurre qui détourne la classe ouvrière de ses véritables combats. Aux débats de l'époque stalinienne sur "le socialisme dans un seul pays" correspondent donc au XIX siècle ceux sur «le socialisme dans un seul village ». Mais la critique morrissienne de l'owenisme ne peut s'appliquer de la même manière à tous les socialistes. Dans Labour's Wrongs, Bray ne prône pas du tout l'instauration d'une communauté autarcique, mais au contraire d'un système de communautés spécialisées (par exemple uniquement sur la production de coton, ou uniquement sur celle de laine), et échangeant entre elles sur des bases égalitaires. Certes, ce n'est pas la prise de pouvoir sur l'ensemble du pays décrite dans News from Nowhere, mais nous avons là une approche systémique plus intéressante que l'utopie owenienne, et moins facilement caricaturable.

\section{Canon, mousquet et mitrailleuse}

14 C'est finalement la question de la lutte des classes qui oppose fondamentalement William Morris à Robert Owen. Dans un autre texte de Commonweal, Morris note: "Depuis l'époque de Robert Owen, la position de la coopération a été complètement bouleversée par l'essor du socialisme révolutionnaire, né de l'application du principe de l'évolution à la société humaine, avec, comme conséquence, la compréhension de la lutte des classes ${ }^{33}$. Owen se voit reprocher d'avoir ignoré les antagonismes de classes, donc d'avoir rêvé un monde sans violence, où la persuasion remplacerait la force physique. Or la violence 
fait partie de la société capitaliste et sera au cœur aussi du processus révolutionnaire. Devant la question de Guest: "Did the change, "the revolution" it used to be called, come peacefully?', Hammond s'exclame : 'Peacefully?... what peace was there amongst those poor confused wretches of the nineteenth century? It was war from beginning to end: bitter war, till hope and pleasure put an end to $i t^{34}$. C'est en ce sens que l'on pourrait dire avec A.L. Morton que Morris a écrit la première « utopie non utopique » ('the first Utopia which is not Utopian' ${ }^{35}$ ).

15 Les owenistes, il est vrai, ont souvent suivi le philanthrope gallois dans son angélisme social. William Thompson ou John Gray ne posent jamais le problème de la violence révolutionnaire. Beaucoup plus stimulante, là encore, me semble la lecture de Labour's Wrongs. Dans les dernières pages de ce texte, que Max Beer a qualifié de «dernier et le plus brillant des manifestes chartistes ${ }^{36}$, Bray développe une réflexion plus complexe et moins idyllique des rapports sociaux. Sur un fond apparemment idéaliste et non violent ('Reason and not force - conviction and not compulsion - purchase and not plunder... are the proper instruments to be employed' ${ }^{37}$ ) point subitement un tout autre discours: 'It is scarcely to be expected that the capitalists, with their present habits and prejudices, will generally consent to sell their property for this object. As a class they will at the moment abhor any transaction which tends, however remotely, to take from their supremacy ${ }^{38}$. Nous voici subitement loin de la douce utopie owenienne et plus proche du climat insurrectionnel du chapitre XVII de News from Nowhere. La réaction de la classe des possédants aux revendications ouvrières se fera, selon Bray, en deux temps. D'abord verbalement: ‘ Many false prophets and interested advisers will rise up and endeavour to mislead and delude the people ${ }^{39}$. Mais très vite, "when vituperation shall have exhausted all its materials in the condemnation of a social change, the weightier arguments of the cannon and the musket will not be far off ${ }^{40}$. Cette évocation du canon et du mousquet préfigure le spectacle apocalyptique de Trafalgar Square, dans News from Nowhere, avec ses mitrailleuses tirant sur la foule : 'It was as if the earth had opened, and hell had come up bodily amidst us... The dead and dying covered the ground, and the shrieks and wails and cries of horror filled all the air, till it seemed as if there was nothing else in the world but murder and death' ${ }^{41}$.

Nous sommes, avec Morris comme avec Bray, dans ce que Morton appellerait «l'utopie anti-utopique ". Pourtant Bray, contrairement à Morris (mais il écrit un demi-siècle plus tôt), n'assume pas les conséquences de ce qu'il vient d'évoquer. La boîte maléfique à peine ouverte, il la referme frileusement pour retrouver la sérénité du philosophe : ‘ Considerations of this character, however, do not concern the inquirer after truth... Individuals have not the power to decide in what manner particular changes shall be accomplished' ${ }^{\prime 2}$. Il faut donc noter que William Morris avait accès à une littérature politique, de langue anglaise, qui intégrait, de façon certes embryonnaire, la lutte de classes dans sa problématique révolutionnaire. Très anti-utopique dans sa prise en compte de l'histoire et des rapports de force au sein de la société, le texte de William Morris n'en demeure pas moins une véritable utopie dans la mesure où le long processus historique débouche sur une véritable "sortie de l'histoire». Sur ce point il rejoint tous les socialistes utopiques britanniques du début du siècle, et les utopies plus anciennes. Ce n'est pas ici le lieu d'étudier l'utopie morrissienne en soi, mais disons simplement que tous les critères de l'utopie classique sont réunis : bonheur universel, harmonie sociale, gel des conflits, refroidissement du politique et de la crise, fin de l'Histoire pour ne pas

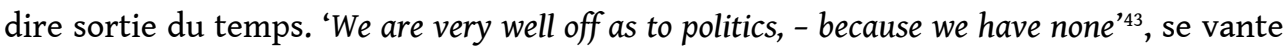
Hammond dans le minuscule chapitre XIII digne de Tristram Shandy. Le sous-titre de 
News from Nowhere, An Epoch of Rest, est révélateur : on est dans le « repos ", la stase, la fin de tout 'political or social unrest'. 'We have been living for a hundred and fifty years, more or less in our present manner', note Hammond ${ }^{44}$. Ce n'est pas la présence, peu convaincante, du 'grumbler', ou la référence à la persistance de crimes passionnels dans le pays de Nulle Part qui change grand chose, et le problème de la «stagnation » et d'une société " figée dans la perfection» n'est pas, contrairement à ce que Paul Meier voudrait nous faire croire, un "faux problème $»^{45}$. Par rapport à ses précurseurs britanniques, Morris est ici dans la continuité plus que dans la rupture.

\section{L'incitation au travail}

17 L'originalité de Morris, son apport le plus précieux à la pensée socialiste, est sans doute sa conception du travail. Ici aussi, sa réflexion prolonge, en l'enrichissant, celle de ses prédécesseurs. Dans le pays de Nulle Part, le travail est devenu un plaisir. 'All work is pleasurable' dit Hammond en soulignant le mot 'all't6. Bien loin est le temps où l'on devait forcer les travailleurs à la tâche. Dick évoque les capitalistes oisifs qui 'in the bad times used to force other people to work for them - the people, you know, who are called slaveholders or employers of labour in the history books ${ }^{47}$. A présent, le travail étant devenu un plaisir, personne ne cherche à l'éviter, au contraire tout le monde le recherche avidement. La frontière entre production de marchandises, artisanat et art s'est estompée et fabriquer une pipe, ou une blague à tabac ${ }^{48}$, par exemple, est à la fois produire un objet utile et créer une œuvre d'art. 'There is conscious sensuous pleasure in the work itself; it is done, that is, by artists ${ }^{49}$. Hammond associe en un nom composé les mots «travail» et "plaisir»: 'the art of work-pleasure, as one ought to call it $t^{50}$. Cette recherche «instinctuelle» du plaisir esthétique, 'a craving for beauty'51, qui s'éveille en l'homme dans la société communiste, est ce qui sauve l'humanité de l'ennui ('a dull level of utilitarian comfort ${ }^{32}$ ) et continue de stimuler les êtres humains lorsqu'ils se sont affranchis de l'oppression et de la coercition.

Car le problème est bien celui de l'incitation au travail dans une société où chacun recevra, non plus «selon son travail » comme dans le stade "socialiste», mais « selon ses besoins ». Si ses besoins sont de toute façon satisfaits, pourquoi se fatiguer ? Un chapitre entier de News from Nowhere est intitulé 'On the lack of incentive to labour in a

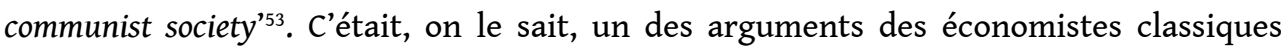
contre les partisans d'une société égalitaire. Pour Malthus, seule la rétribution matérielle pousse l'homme au travail, la retirer serait jeter la société entière dans l'indolence : 'If no man could hope to rise or fear to fall in society; if industry did not bring with it its reward, and indolence its punishment; we could not expect to see that animated activity in bettering our condition which now forms the master-spring of public property'54. C'est à Malthus et aux autres économistes classiques que répond William Morris dans ce chapitre sur l'incitation au travail. Mais il n'est pas le premier, en Grande-Bretagne, à répondre à cet argument. C'est pourtant un socialiste français, Charles Fourier, que Morris cite comme référence: 'Fourier, whom all men laughed at, understood the matter better', note Hammond ${ }^{55}$. C'est chez Fourier que Morris a trouvé cette notion de travail comme plaisir : 'Among these Fourier is the one that calls for most attention, since his doctrine of the necessity and possibility of making labour attractive is one which Socialism can by no means do without ${ }^{356}$. Fourier écrivait : 
Si pourtant l'industrie est la destination qui nous est assignée par le Créateur, comment penser qu'il veuille nous y amener par la violence, et qu'il n'ait pas su mettre en jeu quelque ressort plus noble, quelque amorce capable de transformer les travaux en plaisirs ? ${ }^{57}$

Mais les socialistes britanniques qui ont précédé Morris avaient déjà posé le problème de l'incitation au travail. Eux aussi ont répondu à l'objection malthusienne par une valorisation de l'activité productrice. S'ils n'ont pas élaboré une théorie aussi aboutie que Morris sur le travail-plaisir, ils ont néanmoins avancé des réponses qui sont autant de jalons dans cette direction. Reprenons ce que dit Morris par la bouche de Hammond. Pourquoi le travail est-il devenu plaisir dans le pays de Nulle Part? Trois raisons sont avancées :

'either because of the hope of gain in honour and wealth with which the work is done, which causes pleasurable excitement, even when the actual work is not pleasant'

'or else because it has grown into a pleasurable habit, in the case with what you may call mechanical work'

'and lastly (and most of our work is of this kind) because there is conscious sensuous pleasure in the work itself; it is done, that is, by artists. ${ }^{58}$ pays de Nulle Part), il reste deux facteurs majeurs. Le premier est le « gain d'honneur et de richesse » qu'espère obtenir le travailleur. On sait que Morris distingue 'wealth' et ' riches $^{59}$, et c'est le premier terme, 'wealth', qui est ici employé : richesse au sens noble du terme, à la fois matérielle et morale. Mais ce qui m'intéresse davantage dans cette première raison invoquée, c'est la référence au gain d'honneur. Autrement dit, le plaisir ('pleasurable excitement') que ressent le travailleur, alors même que sa tâche est éventuellement 'not pleasant', vient de l' " honneur » qu'il en tire : honneur par rapport à soi-même, peut-on penser, mais aussi, et peut-être surtout, par rapport aux autres, aux 'neighbours'. Et c'est là que nous retrouvons la raison principale évoquée par les socialistes britanniques du début du siècle pour servir de motivation au travail, dans une société idéale dépourvue de concurrence individuelle, et pour ainsi répondre à l'objection de Malthus.

Ce qui pousse un travailleur à bien faire son travail, dit Bray, c'est avant tout l'estime de ses camarades, qui pèse plus lourd pour lui que le montant de son salaire. L'inventeur, qui travaille jour et nuit tout en restant, en général, dans la pauvreté, ne cherche que cette estime, cet "honneur» dirait Morris. L'opinion est un facteur déterminant dans la société idéale imaginée par les socialistes utopistes du début du siècle. Dans une société communiste, l'opinion publique prendrait un poids beaucoup plus grand: elle acquerrait the energy of family sympathies, of family love, or family disapprobation'60. William Thompson souligne que "we live on the frowns and smiles of our fellow-creatures' ${ }^{\prime 61}$. C'est l'opinion des autres qui est 'the real universal motive to action' ${ }^{\prime 62}$ et donc du travail. La preuve, argumente Thompson, c'est que dans la société actuelle, dans bien des professions le salaire est le même quel que soit l'effort fourni. Pourtant, remarque-t-il, certains travailleurs produisent une fois et demie, voire deux fois plus que leurs compagnons, tout cela pour 'the mere praise of being the best workman'63. Au doux plaisir du regard de l'autre s'ajoute la satisfaction, tout aussi jouissive, du travail bien fait: 'The pleasures of successful industry...: the pleasure of skill, of perseverance, of success $^{\prime 64}$. Bien avant Morris, Thompson associe très intimement travail et plaisir et multiplie, dans les pages sur le travail, les termes 'pleasure', 'accumulated pleasures', 'to heighten the enjoyment'. Pour ressentir ces émotions, il faut, note toutefois Thompson,

Revue Française de Civilisation Britannique, XIII-1 | 2004 
que le travailleur ait la «sécurité » de pouvoir disposer des fruits de son travail ( 'security' est une notion que Thompson emprunte à Bentham).

Le second plaisir lié au travail est celui, que j'ai déjà abordé, de la création : l'activité laborieuse est comparée à l'activité artistique, voire même au plaisir de faire des enfants. Comme le note le vieux Hammond : 'If you are going to ask to be paid for the pleasure of creation, which is what excellence in work means, the next thing we shall hear of will be a bill sent for the begetting of children'65. La création artisanale est donc sacralisée puisque, comme la pro-création, elle donne la vie : 'The reward of labour is life. Is that not enough?'66 Cet argument en faveur du travail " gratuit » est sans doute original, mais on trouve un parallèle intéressant entre production de marchandises et production d'œuvres d'art, dès 1824, chez William Thompson. Dans sa Distribution of Wealth, le socialiste irlandais s'emploie à revaloriser l'activité laborieuse et à stigmatiser l'oisiveté des divers parasites de la société, et il s'en prend au discours qui dénigre les travailleurs: "What more absurd... to pretend to respect wealth produced by labor, and to disdain the labor that produced it? 'Tis admiring Milton's Paradise Lost, and at the same time affecting to despise the intellect that imagined $i t^{67}$. Cette comparaison, assez inattendue il faut bien le dire, entre la production de l'ouvrier et le Paradis perdu de Milton, n'est pas innocente. Même si Thompson ne va pas aussi loin que Morris, il y a en germe dans ce rapprochement l'idée que ce que crée le travail humain est aussi sacré que l'art.

Enfin, on retrouve chez les premiers socialistes du Royaume-Uni le rejet de la production de marchandises inutiles, qui est l'un des leitmotivs de News from Nowhere. Thompson n'a que mépris pour tous ces objets inutiles que les riches n'achètent que pour se distinguer: 'baubles', 'costly trinkets', 'all things that serve for mere distinction beyond their intrinsic value', qui ne sont pour lui que 'the fool's pursuit' ${ }^{\prime}$. Pourtant, comme Morris plus tard, Thompson n'exclut pas les objets inutiles mais beaux, à condition que tous les biens nécessaires aient déjà été acquis par tous. Quand rien d'essentiel ne manquera, 'then and not before, would the unemployed time of such a community, be as harmlessly devoted to the gathering and polishing of pretty stones as to the cultivating of ornamental pinks and roses ${ }^{169}$. Cet encouragement à la production de pierres précieuses et de fleurs annonce la société du pays de Nulle Part. Mais cette production n'aurait rien à voir avec le luxe inutile du système commercial. On pense aux attaques de Morris contre le XIX ${ }^{e}$ siècle britannique dont la préoccupation principale ('the chief business') est de fabriquer des choses dont personne ne veut ('making things that nobody wants' ${ }^{70}$ ), produisant 'in a never ending series sham or artificial necessaries" quantities of worthless makeshifts" ${ }^{72}$.

\section{Conclusion}

Grande est la tentation d'étudier William Morris dans le contexte des vingt dernières années de son siècle et de lui prêter les sources que lui-même choisit de nommer: Marx, Ruskin, Carlyle, Fourier, par exemple. Cet article ne cherche bien sûr pas à minimiser ces influences, ni même à vouloir établir une filiation directe entre l'auteur de News from Nowhere et les premiers socialistes britanniques du début du siècle. Il souhaite simplement rappeler qu'avant la traversée du désert - au milieu du siècle - de la pensée socialiste britannique, il y avait une effervescence idéologique qui ne se résume pas au seul owenisme, et que cette production riche et plurielle a imprégné le mouvement ouvrier, au travers de ses militants et de leurs débats. William Morris se 
trouve, de fait, au bout de ce long chemin et hérite en partie, consciemment ou non, de ces forces endogènes. Il n'est jamais de rupture totale dans l'histoire des idées, et dans l'histoire de la pensée socialiste en particulier, et le voyageur utopien qu'inventa Bray lorsqu'il écrivit, au cours de l'hiver 1840-41, son Voyage from Utopia to Several Unknown Regions of the World, ${ }^{73}$ ce voyageur ne se sentirait pas trop dépaysé si les hasards de sa navigation le faisaient débarquer, un beau jour du XXII siècle, dans le pays de Nulle Part, l'Angleterre rêvée par William Morris.

\section{NOTES}

1. E.P. THOMPSON, William Morris : Romantic to Revolutionary, London: Lawrence \& Wishart, 1955 (New York: Pantheon Books, 1955, p. 13).

2. Paul MEIER, La Pensée utopique de William Morris, Paris : Éditions sociales, 1972.

3. On parle également des "owenistes", terme qui pose lui aussi problème, ou, pour certains d'entre eux, de «socialistes ricardiens » en référence à la théorie de la valeur-travail de David Ricardo.

4. Voir J.E. KING, 'Utopian or Scientific ? A Reconsideration of the Ricardian Socialists', History of Political Economy, XV, 3, 1983, p. 345.

5. Thomas CARLYLE, Chartism (1839), in Thomas CARLYLE, Selected Writings, Harmondsworth: Penguin, 1971, p. 219.

6. E.P. THOMPSON, op .cit., p. 277.

7. François BÉDARIDA, «Le socialisme en Grande-Bretagne de 1875 à 1914 », in Jacques DROZ (dir.), Histoire générale du socialisme, t.2, De 1875 à 1918, Paris : PUF, 1974, p. 347.

8. Ibid., p. 348.

9. Ibid., p. 348.

10. Thomas COOPER, The Life of Thomas Cooper (1872) (Leicester: Leicester University Press, 1971, p. 394).

11. J'emploie ici l'adjectif «britanniques » par commodité. Si Hodgskin était bien anglais et Gray écossais, Thompson était irlandais et Bray, bien que résidant à Leeds durant deux décennies et étant pris pour un Anglais par plusieurs historiens, était américain.

12. Le livre de MORRIS et BAX, Socialism, its Growth and Outcome (1893) cite Misère de la philosophie. Marx qualifie Labour's Wrongs de "remarquable" avant de le citer très longuement. Voir, K. Marx, Misère de la philosophie : réponse à la Philosophie de la misère de M. Proudhon, Paris : A. Frank, 1847 (Paris : Éditions sociales, 1972, pp. 79-89).

13. Paul MEIER, op. cit., p. 243.

14. Ibid., p. 408.

15. Ibid., p. 408.

16. Cité et traduit par MEIER, op.cit., p. 428.

17. William MORRIS, News from Nowhere, Clive WILMER (ed.), London: Penguin, 1998, p.157.

18. Ibid., p. 187.

19. Ibid., p. 158.

20. Ibid., p. 162.

21. John Francis BRAY, Labour's Wrongs and Labour's Remedy, or the Age of Might and the Age of Right, Leeds: David Green, 1839, p. 134. 
22. Ibid., p. 134.

23. „Das Ensemble der gesellschaftlichen Verhältnisse“.

24. William MORRIS, News from Nowhere, p. 118.

25. J.F. BRAY, op. cit., p.113.

26. Ibid., p.111.

27. William MORRIS, News from Nowhere, p. 145.

28. J.F. BRAY, op.cit., p. 99.

29. Ibid., p.89. C'est moi qui souligne.

30. A.L. MORTON, Socialism in Britain, London: Lawrence \& Wishart, 1963, pp. 30-31.

31. Paul MEIER, op.cit., p. 271.

32. Cité et traduit par MEIER, op.cit., p. 274.

33. Cité et traduit par MEIER, ibid., p. 273.

34. W. MORRIS, News from Nowhere, p. 133.

35. Cité par E.P. THOMPSON, op.cit., p. 697.

36. „Das letzte und glänzendste Manifest des Owenismus“. Max BEER, „Die owenistisch-chartistische Periode und J.F. Bray“, in J.F. BRAY, Die Leiden der Arbeiterklasse und ihr Heilmittel. Eingeleitet und übers. von M. Beer. Leipzig : C.L. Hirschfeld, 1920, p. 1.

37. J.F. BRAY, Labour's Wrongs and Labour's Remedy, p. 215.

38. Ibid., p. 216.

39. Ibid., p. 216.

40. Ibid., p. 216.

41. W. MORRIS, News from Nowhere, p. 144.

42. J.F. BRAY, op.cit., p. 216.

43. W. MORRIS, News from Nowhere, p. 116.

44. Ibid., p. 111.

45. P. MEIER, op.cit., p. 823.

46. Ibid., p. 122.

47. W. MORRIS, News from Nowhere, p. 75.

48. Voir W. MORRIS, News from Nowhere, chapitre VI, pp. 70 seq.

49. Ibid., p. 123.

50. Ibid., p. 160.

51. Ibid., p. 160.

52. Ibid., p. 159.

53. Ibid., chapitre XV, pp. 121 seq.

54. T.R. MALTHUS, An Essay on the Principle of Population (1798) (Bungay: Everyman, 1973, p. 254).

55. W. MORRIS, News from Nowhere, p. 122.

56. W. MORRIS, 'The Hopes of Civilization', op.cit., p. 322.

57. Cité par P. MEIER, op.cit., p. 262.

58. W. MORRIS, News from Nowhere, pp. 122-3. C'est bien sûr moi qui découpe.

59. On se rappelle la formule concernant les habitants de Nulle Part, 'who had cast away riches and attained to wealth', W. MORRIS, News from Nowhere, p. 219.

60. William THOMPSON, An Inquiry into the Principles of the Distribution of Wealth, Most Conducive to Human Happiness (1824) (New York: Kelley, 1963, p. 395).

61. Ibid., p. 468.

62. Ibid., p. 469.

63. Ibid., p. 470.

64. Ibid., p. 61.

65. W. MORRIS, News from Nowhere, p. 122.

66. Ibid., p. 122.

67. William THOMPSON, op.cit., p. 89. 
68. Ibid., pp. 399- 400.

69. Ibid., p. 400.

70. W. MORRIS, News from Nowhere, p. 107.

71. Ibid., p. 124.

72. Ibid., p. 126.

73. J.F. BRAY, A Voyage from Utopia to Several Unknown Regions of the World (1840-41). London: Lawrence \& Wishart, 1957.

\section{RÉSUMÉS}

Si l'influence de Marx a fait l'objet de nombreuses études, dont la fameuse biographie de Paul Meier, les liens entre les Nouvelles de Nulle Part et les écrits des premiers socialistes britanniques n'ont pas été suffisamment explorés. Cet article souhaite relier les deux périodes d'intense activité que sont, pour la pensée socialiste, le début et la fin du XIX ${ }^{\mathrm{e}}$ siècle. L'analyse de l'œuvre de deux socialistes, John Francis Bray et William Thompson, se propose de dégager d'éventuelles connexions, par exemple à propos de la théorie des deux phases de la révolution, du rôle moteur du prolétariat ou de la question de l'incitation au travail dans la société communiste.

Though the Marxist influence on Morris's News from Nowhere has often been emphasised, especially by Paul Meier in his famous biography, little has been written about possible connections between two highly productive periods in the $19^{\text {th }}$ century: the 1820 s and 1830 s on the one hand, and the late 1880s and 1890s on the other. An analysis of two socialist authors, John Francis Bray and William Thompson, shows some possible links on such topics as the twostage theory of the Revolution, the role of the working class or the question of incentives to work in a communist society.

\section{AUTEUR}

\section{MICHEL PRUM}

Université Denis Diderot Paris 7 